\title{
改善土壤通气性促进甘薯源库间光合产物运转的原因解析
}

\author{
刘永晨 ${ }^{1}$ 司成成 ${ }^{2}$ 柳洪鹃 ${ }^{1, *}$ 张涁涁 ${ }^{1}$ 史春余 ${ }^{1, *}$ \\ ${ }^{1}$ 山东农业大学农学院 / 作物生物学国家重点实验室, 山东泰安 $271018 ;{ }^{2}$ 海南大学园艺学院, 海南海口 570228
}

摘 要: 为了明确土壤通气性对甘薯源库间光合产物运转的调控机制, 本研究以淀粉型品种商薯 19 和济徐 23 为试验材 料, 设置疏松、对照和紧实 3 个处理进行大田试验, 研究结果表明, 与对照处理相比, 疏松处理显著提高 2 个品种的块 根产量和经济系数, 2 年平均增幅分别为 $27.03 \%$ 38.74\%和 $6.30 \%$ 13.05\%, 紧实处理则显著降低 2 个品种的块根产量和 经济系数, 2 年平均降幅分别为 $17.87 \%$ 15.92\%和 $10.83 \% \sim 15.63 \%$ 。功能叶 ${ }^{13} \mathrm{C}$ 标记结果显示, 疏松处理显著提高块根中 光合产物的输入效率。疏松处理显著提高块根中蔗糖和淀粉含量, 显著降低地上部器官中淀粉含量和茎中尤其是茎的中 下部中蔗糖含量; 紧实处理则显著降低块根中蔗糖和淀粉含量, 而显著提高地上部器官蔗糖和淀粉含量, 且茎中下部 蔗糖含量增幅较大。疏松处理显著降低 50 150 d 茎基部与茎顶部间和茎基部与块根间的蔗糖含量差; 紧实处理则显著 提高茎基部与茎顶部间和茎基部与块根间的蔗糖含量差，且茎基部与块根间蔗糖含量差的变幅大于茎基部与茎顶部间 的蔗糖含量差。相关分析表明, 茎基部与块根间、茎基部与茎顶部间蔗糖含量差与块根蔗糖和淀粉含量呈极显著负相关。 说明改善土壤通气性可促进茎基部光合产物向块根的运转, 提高块根中碳水化合物含量, 增加块根产量。

关键词：甘薯; 土壤通气性；块根产量；光合产物；运转

\section{Reason exploration for soil aeration promoting photosynthate transportation between sink and source in sweet potato}

\author{
LIU Yong-Chen ${ }^{1}$, SI Cheng-Cheng ${ }^{2}$, LIU Hong-Juan ${ }^{1, *}$, ZHANG Bin-Bin ${ }^{1}$, and SHI Chun-Yu ${ }^{1, *}$ \\ ${ }^{1}$ College of Agronomy, Shandong Agricultural University / State Key Laboratory of Crop Biology, Tai'an 271018, Shandong, China; ${ }^{2}$ College of \\ Horticulture and Landscape Architecture, Hainan University, Haikou 570228, Hainan, China
}

\begin{abstract}
Field experiments were performed using the varieties of starchy sweet potato Shangshu 19 and Jixu 23 with three treatments including loose soil, control soil and compact soil, to clarify the regulatory mechanism of soil compaction on transportation of photosynthates between sink and source of sweet potato. Compared with control treatment, storage root yield and economic coefficient of loose soil treatment were significantly increased by $27.03 \%-38.74 \%$ and $6.30 \%-13.05 \%$ in two years, respectively, while those of compact soil treatment significantly decreased by $17.87 \%-15.92 \%$ and $10.83 \%-15.63 \%$, respectively. The ${ }^{13} \mathrm{C}$ labeling results of functional leaves showed that loose treatment significantly improved the import efficiency of photosynthate in storage roots. Loose soil treatment significantly increased sucrose and starch contents in storage roots, but significantly reduced starch content in aboveground organs, especially in lower-middle position of stem. Compact soil treatment significantly decreased sucrose and starch contents in storage roots, but significantly increased starch and sucrose contents in aboveground organs especially in lower-middle position of stem. Both difference of sucrose content between stem base and stem top and between stem base and storage root at 50-150 days after planting in loose treatment were significantly decreased. While, significantly increased in compact treatment. The variation range of sucrose content difference between stem base and storage root was larger than between stem base and stem top. There was a very significantly negative correlation between the sucrose content difference of stem base and storage root, and the sucrose and starch content in storage root. Improvement of soil aeration, can promote the transportation of photosynthates from stem base to storage root, increase carbohydrate content in storage root and enhance storage root yield.
\end{abstract}

本研究由国家自然科学基金项目(31371577, 31701357)和山东省薯类产业创新团队首席专家项目(SDAIT-16-01)资助。

This study was supported by the National Natural Science Foundation of China $(31371577,31701357)$ and the Potato Innovation Program for Chief Expert of Shandong Province (SDAIT-16-01).

* 通信作者(Corresponding authors): 史春余, E-mail: scyu@sdau.edu.cn, Tel: 0538-8246259; 柳洪鹃, E-mail: liumei0535@126.com

第一作者联系方式: E-mail: liuyongchensdau@163.com

Received (收稿日期): 2019-03-08; Accepted (接受日期): 2019-09-26; Published online (网络出版日期): 2019-10-12.

URL: http://kns.cnki.net/kcms/detail/11.1809.S.20191012.1214.004.html 
Keywords: sweet potato; soil aeration; storage root yield; photosynthate; transportation

甘薯用途广, 适应性强 ${ }^{[1-4]}$, 丘陵山地多有种植 ${ }^{[5-6]}$ 。 近年来, 随着甘薯生产收益的增加, 甘薯种植也不断向平 原地区扩张 ${ }^{[7]}$ 。甘薯是以地下块根为收获物, 土壤疏松、 通气良好是其获得高产的主要土壤条件之一 ${ }^{[8-9]}$ 。土壤黏 度大、板结、通气性差时, 块根产量降低、品质变差。丘 陵山地因水浇条件差, 土壤极易因干旱而板结; 平原地区 随着机械化的普及和肥料的不合理施用, 耕作层土壤的 紧实度逐年增加 ${ }^{[10-12]}$, 土壤通气状况日益恶化, 严重影 响甘薯块根产量。土壤通气状况已经成为制约甘薯高产稳 产的主要因素之一。

改善土壤通气性可以促进块根膨大过程中光合产物由 地上部向块根的运输, 提高干物质在块根中的分配率, 显 著提高甘薯块根产量 ${ }^{[13-15]}$ 。关于土壤通气性调控光合产物 运转分配的生理原因, 前人进行了有益的探索, 一般认为 改善通气性可以提高块根形成层的活动能力 ${ }^{[16]}$, 增加块根 中 ATP 含量和脱落酸(ABA)含量 ${ }^{[17]}$, 降低淀粉酶活性 ${ }^{[14-15]}$ 等促进源库间光合产物运转。但前人的研究多集中于块根 的膨大特性, 而对光合产物由叶片装载、经茎运输后卸载 到块根的整个运转过程的关注较少, 缺乏对土壤通气性调 控光合产物运转关键环节的认识。因此, 本研究设置不同 通气性的土壤条件, 从源库间光合产物运转效率、不同器 官和功能叶以下茎不同部位蔗糖和淀粉含量、不同器官间 蔗糖含量差等方面系统分析土壤通气性引起光合产物由源 到库运转差异的关键环节, 研究结果可以为促进甘薯源库 间光合产物运转及栽培措施的改进提供理论依据。

\section{1 材料与方法}

\section{1 试验材料与设计}

2017 年和 2018 年在山东农业大学农学试验站进行大 田试验。供试品种为商薯 19 和济徐 23 , 供试土壤为沙壤 土。2017 年 $0 \sim 20 \mathrm{~cm}$ 土层含碱解氮 $106.29 \mathrm{mg} \mathrm{kg}^{-1}$ 、速效 磷 $36.93 \mathrm{mg} \mathrm{kg}^{-1}$ 、速效钾 $109.67 \mathrm{mg} \mathrm{kg}^{-1}$ 、有机质 $0.97 \%$ 。 2018 年 $0 \sim 20 \mathrm{~cm}$ 土层含碱解氮 $88.82 \mathrm{mg} \mathrm{kg}^{-1}$ 、速效磷 $33.27 \mathrm{mg} \mathrm{kg}^{-1}$ 、速效钾 $90.35 \mathrm{mg} \mathrm{kg}^{-1}$ 、有机质 $1.05 \%$ 。依 据土壤容重 ${ }^{[18]}$ 和紧实度设置 3 个处理: (1)紧实区 (JS), 压 实试验地而成, $0 \sim 20 \mathrm{~cm}$ 土层的容重为 $1.40 \sim 1.50 \mathrm{~g} \mathrm{~cm}^{-3}$, 紧实度大于 $600 \mathrm{kPa}$ ，一般不高于 $1200 \mathrm{kPa}$ 。(2)标准区 (CK), 0 20 cm 土层的容重为 $1.30 \sim 1.40 \mathrm{~g} \mathrm{~cm}^{-3}$, 紧实度为 300 400 kPa。(3)疏松区(SS), 试验地土壤、有机肥和沙混 合而成, 其土壤有机质含量和标准区一致并将 3 个处理的 速效氮、磷、钾含量调到基本一致, $0 \sim 20 \mathrm{~cm}$ 土层的容重 为 $1.20 \sim 1.30 \mathrm{~g} \mathrm{~cm}^{-3}$, 紧实度为 $100 \sim 200 \mathrm{kPa}$ 。3 个处理土 壤物理性状如表 1 所示。采取裂区试验设计, 品种为主区, 紧实度为副区, 重复 3 次, 小区面积为 $10 \mathrm{~m}^{2}$, 行距 $80 \mathrm{~cm}$, 株距 $25 \mathrm{~cm}$ 。在栽种期、块根膨大前期、块根膨大高峰期、 块根膨大后期用土壤紧实度仪测量土壤紧实度。栽积后间 隔 $5 \mathrm{~d}$ 测量土壤 $0 \sim 20 \mathrm{~cm}$ 和 20 40 $\mathrm{cm}$ 土层土壤体积含水 量。小区每平方米含水量 $\left(\mathrm{m}^{3}\right)$ 为 $\mathrm{H} \times 1 \mathrm{~m}^{2} \times$ 土壤体积含水量 (\%), 其中 $\mathrm{H}$ 为土层深度。以含水量最高的小区为标准将 其余小区含水量补齐。

\section{表 1 栽种期土壤物理性状}

Table 1 Physical characteristics of the soil in planting

\begin{tabular}{|c|c|c|c|c|c|c|c|c|}
\hline $\begin{array}{l}\text { 年份 } \\
\text { Year }\end{array}$ & $\begin{array}{c}\text { 处理 } \\
\text { Treatment }\end{array}$ & $\begin{array}{c}\text { 土层深度 } \\
\text { Soil layer } \\
\quad(\mathrm{cm})\end{array}$ & $\begin{array}{c}\text { 土壤紧实度 } \\
\text { Soil volume } \\
\text { weight }(\mathrm{kPa})\end{array}$ & $\begin{array}{c}\text { 土壤容重 } \\
\text { Soil } \\
\text { compactness } \\
\left(\mathrm{g} \mathrm{cm}^{-3}\right)\end{array}$ & $\begin{array}{l}\text { 土壤比重 } \\
\text { Soil specific } \\
\text { gravity } \\
\left(\mathrm{g} \mathrm{cm}^{-3}\right)\end{array}$ & $\begin{array}{c}\text { 总孔隙度 } \\
\text { Total } \\
\text { porosity } \\
(\%)\end{array}$ & $\begin{array}{c}\text { 毛管孔隙度 } \\
\text { Capillary } \\
\text { porosity (\%) }\end{array}$ & $\begin{array}{c}\text { 非毛管孔隙度 } \\
\text { Non-capillary } \\
\text { porosity } \\
(\%)\end{array}$ \\
\hline \multirow[t]{6}{*}{2017} & SS & $5-10$ & 126.49 & 1.26 & 2.58 & 51.35 & 24.86 & 26.49 \\
\hline & & $15-20$ & 224.23 & 1.30 & 2.57 & 49.35 & 25.50 & 23.85 \\
\hline & $\mathrm{CK}$ & $5-10$ & 301.16 & 1.33 & 2.64 & 49.81 & 24.15 & 25.66 \\
\hline & & $15-20$ & 464.12 & 1.39 & 2.73 & 49.19 & 31.68 & 17.51 \\
\hline & JS & $5-10$ & 541.63 & 1.46 & 2.73 & 46.38 & 31.78 & 14.60 \\
\hline & & $15-20$ & 927.74 & 1.49 & 2.75 & 45.71 & 38.30 & 7.41 \\
\hline \multirow[t]{6}{*}{2018} & SS & $5-10$ & 143.17 & 1.25 & 2.57 & 50.69 & 24.62 & 26.07 \\
\hline & & $15-20$ & 174.17 & 1.29 & 2.58 & 49.62 & 25.46 & 24.16 \\
\hline & CK & $5-10$ & 267.91 & 1.33 & 2.65 & 48.92 & 25.30 & 23.62 \\
\hline & & $15-20$ & 508.06 & 1.38 & 2.73 & 48.65 & 30.14 & 18.51 \\
\hline & JS & $5-10$ & 826.07 & 1.47 & 2.74 & 46.66 & 31.21 & 15.45 \\
\hline & & $15-20$ & 1230.60 & 1.49 & 2.75 & 45.83 & 36.91 & 8.92 \\
\hline
\end{tabular}

SS：疏松土壤; CK：对照土壤; JS：紧实土壤。

SS: loose soil; CK: control soil; JS: compact soil. 


\section{2 测定项目与方法}

\subsection{1 收获期测产 在收获期测定每个小区每行的薯} 块数和块根鲜重。

1.2.2 甘薯各器官中碳水化合物含量 自栽后 $50 \mathrm{~d}$ 开 始取样, 每隔 $20 \mathrm{~d}$ 取样一次, 共计取样 6 次。留样时选 取各品种每个处理长势基本一致的植株 5 株, 分为叶 片、叶柄、茎蔓及块根四部分。叶片和叶柄各 $200 \mathrm{~g}$; 块 根切片混匀后留取 $200 \mathrm{~g}$; 茎蔓中的主茎留取茎顶部、

茎中部和茎基部三部分样品, 茎顶部为主茎倒 5 叶叶柄 所在位置向下延伸 $10 \sim 15 \mathrm{~cm}$, 茎中部为主茎(去掉自生 长点向下第 5 片展开叶以上部分)对折后, 自中点向两 侧延伸 5.0 7.5 cm, 茎基部为主茎最下面 10 15 cm (图 1), 将主茎剩余部分和侧茎剪碎混匀后留取 $200 \mathrm{~g}$ 。以 上样品 $105^{\circ} \mathrm{C}$ 杀青, $60^{\circ} \mathrm{C}$ 烘干, 磨成粉末过篮, 用于蔗 糖和淀粉含量的测定。采用葸酮比色法测定蔗糖和淀粉 含量 ${ }^{[19]}$ 。

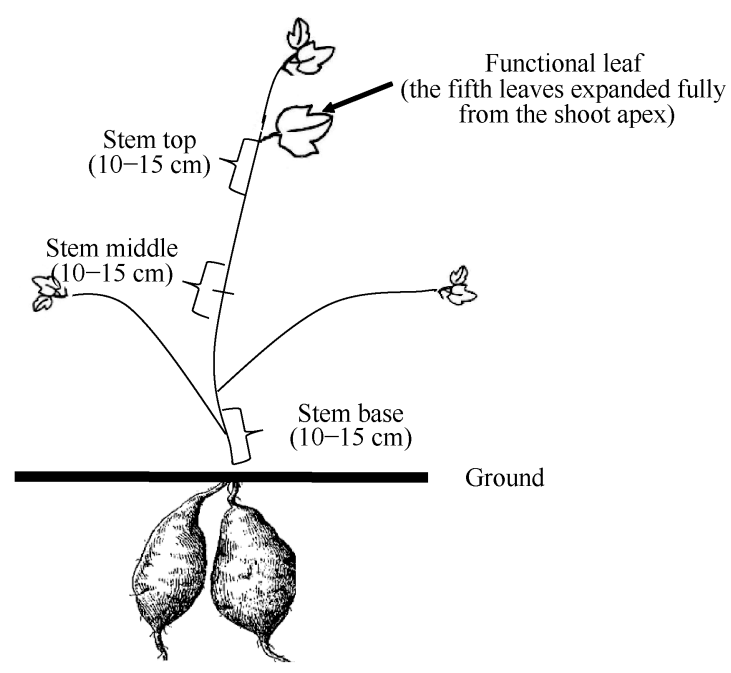

图 1 主茎各部位划分模式图

Fig. 1 Patterns of main stem partition

\subsection{3 经济系数 块根鲜重与整株鲜重的比值。}

1.2.4 器官间庶糖含量差均以前面器官的蔗糖含量 作为被减数, 计算公式如下:

柄与叶间 $(\%)=($ 柄 - 叶 $) /[($ 柄 + 叶 $) / 2] \times 100$

柄与茎顶间 $(\%)=($ 柄-茎顶 $) /[($ 柄 + 茎顶 $) / 2] \times 100$

茎基部与茎顶部间 $(\%)=($ 基部 - 顶部 $) /[$ ( 基部 + 顶 部) $/ 2] \times 100$

茎基部与块根间 (\%)=(基部 - 块根 $) /[($ 基部 + 块 根) $/ 2] \times 100$ 。

1.2.5 ${ }^{13} \mathrm{C}$ 标记于块根膨大中期(栽秧后 $100 \mathrm{~d}$ 左右), 选择晴朗无风天气 9:00-11:00, 从每个小区选择生长基本
一致、具有代表性的植株 3 株, 在其主茎顶部第 4 片和第 5 片展开叶上标记 ${ }^{13} \mathrm{CO}_{2}$ 。由 $\mathrm{Ba}^{13} \mathrm{CO}_{3}\left(99 \%{ }^{13} \mathrm{C}\right)$ 和磷酸在反 应器中生成 ${ }^{13} \mathrm{CO}_{2}$, 并用气球收集; 标记前将欲标记叶用 体积约为 $400 \mathrm{~mL}$ 的聚氯乙烯透明塑料薄膜袋密封, 用医 用注射器注入 $50 \mathrm{~mL}^{13} \mathrm{CO}_{2}(1 \%)$; 光合同化 $40 \mathrm{~min}$, 之后 撤掉塑料薄膜袋。标记完成 $24 \mathrm{~h}$ 后, 剪取植株地上部, 挖 出地下部块根。主要留取: 块根、标记叶及其所在的标 记茎和标记柄、主茎、侧茎、侧叶。分样后将块根切片, 茎切段, 装袋, 经 $105^{\circ} \mathrm{C}$ 杀青 $10 \sim 30 \mathrm{~min}$, 在 $60^{\circ} \mathrm{C}$ 烘箱中 烘干至恒重; 然后称重、粉碎, 用质谱仪(Isoprime 100) 测定 $\delta^{13} \mathrm{C}$ 。

\section{3 数据处理与分析}

采用 Microsoft Excel 2007 分析数据和作图, 统计分 析中的方差分析检验采用 DPS (Data Processing System) v7.05 数据处理系统。采用 SPSS Statistics 20 进行相关性 分析。

\section{2 结果与分析}

\section{1 块根产量及经济系数}

由表 2 可知, 与对照处理相比, 疏松处理显著提高两 品种的块根产量和经济系数, 2 年平均增幅分别为 $27.03 \% \sim 38.74 \%$ 和 $6.30 \% \sim 13.05 \%$, 紧实处理则显著降低 块根产量和经济系数，2 年平均降幅分别为 $17.87 \%$ $15.92 \%$ 和 $10.83 \% \sim 15.63 \%$ 。疏松处理的单薯重显著高于对 照, 而紧实处理则显著低于对照。即疏松处理主要通过提 高单薯重, 提高经济系数而增产, 紧实处理则因降低单薯 重, 降低了经济系数而减产。

\section{2 功能叶 ${ }^{13} \mathrm{C}$ 的分配率和运转效率}

由表 3 可知, 块根快速膨大期, 与对照处理相比, 疏 松处理显著提高块根中 ${ }^{13} \mathrm{C}$ 同化物的分配率, 显著降低其 他器官 ${ }^{13} \mathrm{C}$ 同化物分配率; 紧实处理与之相反。 ${ }^{13} \mathrm{C}$ 标记 后 $24 \mathrm{~h}$ 后, 与对照处理相比, 疏松处理显著提高块根中 ${ }^{13} \mathrm{C}$ 丰度单位时间增加量, 显著降低标记叶中 ${ }^{13} \mathrm{C}$ 丰度单 位时间降低量和主茎 ${ }^{13} \mathrm{C}$ 丰度单位时间增加量; 紧实处理 变化规律与之相反。

\section{3 不同器官蔗糖和淀粉含量}

由表 4 可知, 与对照处理相比, 疏松处理 2 个品种叶 片、叶柄和块根中蔗糖含量显著提高, 而茎中蔗糖含量显 著降低; 紧实处理则与之相反, 两年数据规律一致。由表 5 可知, 疏松处理块根中淀粉含量显著高于对照处理, 紧 实处理则显著低于对照处理; 疏松处理叶、柄和茎中淀粉 含量分别自栽秧后 $90 \mathrm{~d} 、 70 \mathrm{~d}$ 和 $50 \mathrm{~d}$ 至收获期均显著低 于对照处理，而紧实处理则显著高于对照处理。即改善土 壤通气性块根中蔗糖和淀粉快速积累开始的早、积累时间 长; 降低土壤通气性茎部蔗糖和淀粉的积累效率高。 
表 2 块根产量及经济系数

Table 2 Yield of storage root and its economic coefficient

\begin{tabular}{|c|c|c|c|c|c|c|}
\hline $\begin{array}{l}\text { 年份 } \\
\text { Year }\end{array}$ & $\begin{array}{c}\text { 品种 } \\
\text { Variety }\end{array}$ & $\begin{array}{c}\text { 处理 } \\
\text { Treatment }\end{array}$ & $\begin{array}{c}\text { 单株结薯数 } \\
\text { Number of storage root } \\
\text { (lump plant }^{-1} \text { ) }\end{array}$ & $\begin{array}{c}\text { 单薯重 } \\
\text { Fresh weight } \\
\left(\mathrm{g} \mathrm{lump}^{-1}\right)\end{array}$ & $\begin{array}{c}\text { 块根产量 } \\
\text { Storage root yield } \\
\left(\mathrm{kg} \mathrm{hm}^{-2}\right) \\
\end{array}$ & $\begin{array}{c}\text { 经济系数 } \\
\text { Economic coefficient } \\
(\%) \\
\end{array}$ \\
\hline \multirow[t]{6}{*}{2017} & 商薯 19 & SS & $4.56 \mathrm{~b}$ & $244.31 \mathrm{a}$ & $55660.87 \mathrm{a}$ & $70.47 \mathrm{a}$ \\
\hline & Shangshu 19 & $\mathrm{CK}$ & $4.42 \mathrm{~b}$ & $214.53 \mathrm{~b}$ & $47280.21 \mathrm{~b}$ & $65.90 \mathrm{~b}$ \\
\hline & & JS & $5.72 \mathrm{a}$ & $141.49 \mathrm{c}$ & $40206.75 \mathrm{c}$ & $61.79 \mathrm{c}$ \\
\hline & 济徐 23 & SS & $3.58 \mathrm{~b}$ & $315.09 \mathrm{a}$ & $55913.79 \mathrm{a}$ & $79.61 \mathrm{a}$ \\
\hline & Jixu 23 & $\mathrm{CK}$ & $3.47 \mathrm{~b}$ & $274.83 \mathrm{~b}$ & $46897.60 \mathrm{~b}$ & $75.34 \mathrm{~b}$ \\
\hline & & JS & $4.39 \mathrm{a}$ & $188.87 \mathrm{c}$ & $41110.64 \mathrm{c}$ & $63.72 \mathrm{c}$ \\
\hline \multirow[t]{6}{*}{2018} & 商薯 19 & SS & $3.99 \mathrm{~b}$ & $296.34 \mathrm{a}$ & $59113.79 \mathrm{a}$ & $80.13 \mathrm{a}$ \\
\hline & Shangshu 19 & $\mathrm{CK}$ & $3.88 \mathrm{~b}$ & $223.33 \mathrm{~b}$ & $43361.92 \mathrm{~b}$ & $67.57 \mathrm{~b}$ \\
\hline & & JS & $4.62 \mathrm{a}$ & $148.89 \mathrm{c}$ & $34353.97 \mathrm{c}$ & $56.35 \mathrm{c}$ \\
\hline & 济徐 23 & SS & $2.99 \mathrm{~b}$ & $363.18 \mathrm{a}$ & $54170.01 \mathrm{a}$ & $89.68 \mathrm{a}$ \\
\hline & Jixu 23 & CK & $2.91 \mathrm{~b}$ & $243.25 b$ & $34227.44 \mathrm{~b}$ & $83.42 \mathrm{~b}$ \\
\hline & & JS & $3.56 \mathrm{a}$ & $154.82 \mathrm{c}$ & $27550.52 \mathrm{c}$ & $71.19 \mathrm{c}$ \\
\hline
\end{tabular}

标以不同字母的值在处理间差异显著 $(P<0.05)$ 。缩写同表 1 。

Values followed by different letters within the same column are significantly different among different treatments at the 0.05 probability level. Abbreviations are the same as those given in Table 1 .

表 3 块根快速膨大期各器官内 ${ }^{13} \mathrm{C}$ 同化物的分配率 $(\%, 2017$ 年, 品种为商薯 19$)$

Table 3 Distribution rate of ${ }^{13} \mathrm{C}$ assimilate in different organs during storage root expanding period (\%, 2017, Variety: Shangshu 19)

\begin{tabular}{cccccccc}
\hline $\begin{array}{c}\text { 处理 } \\
\text { Treatment }\end{array}$ & $\begin{array}{c}\text { 块根 } \\
\text { Storage root }\end{array}$ & $\begin{array}{c}\text { 侧茎 } \\
\text { Side stem }\end{array}$ & $\begin{array}{c}\text { 侧叶和生长点 } \\
\text { Side leaf and growing } \\
\text { point }\end{array}$ & $\begin{array}{c}\text { 主茎 } \\
\text { Main stem }\end{array}$ & $\begin{array}{c}\text { 标叶 } \\
\text { Labeled leaf }\end{array}$ & $\begin{array}{c}\text { 标茎 } \\
\text { Labeled stem }\end{array}$ & $\begin{array}{c}\text { 标唡 } \\
\text { Labeled petiole }\end{array}$ \\
\hline SS & 53.62 & 15.57 & 27.18 & 2.91 & 0.39 & 0.19 & 0.15 \\
CK & 49.69 & 17.24 & 27.63 & 4.07 & 0.82 & 0.28 & 0.26 \\
JS & 44.75 & 17.47 & 31.12 & 5.17 & 0.85 & 0.27 & 0.38 \\
\hline
\end{tabular}

标以不同字母的值在处理间差异显著 $(P<0.05)$ 。缩写同表 1 。

Values followed by different letters in the same column are significantly different among different treatments at the 0.05 probability level. Abbreviations are the same as those given in Table 1.

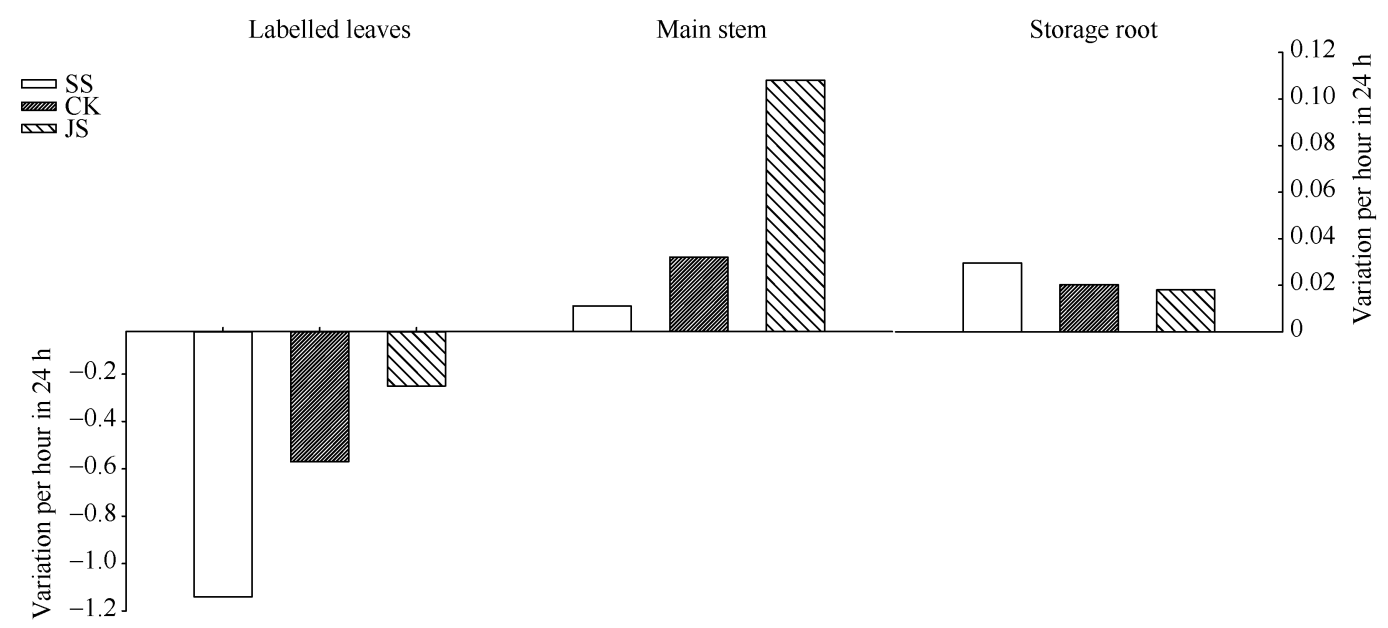

图 2 标记叶、主茎和块根中 ${ }^{13} \mathrm{C}$ 丰度变化特点(2017 年)

Fig. 2 Abundance changes of ${ }^{13} \mathrm{C}$ in leaves, main stems and storage root (2017)

缩写同表 1。Abbreviations are the same as those given in Table 1. 
表 4 不同器官蔗糖含量

Table 4 Sucrose content in different organs (\%)

\begin{tabular}{|c|c|c|c|c|c|c|c|c|c|}
\hline \multirow{2}{*}{$\begin{array}{l}\text { 年份 } \\
\text { Year }\end{array}$} & \multirow{2}{*}{$\begin{array}{l}\text { 器官 } \\
\text { Organ }\end{array}$} & \multirow{2}{*}{$\begin{array}{c}\text { 品种 } \\
\text { Variety }\end{array}$} & \multirow{2}{*}{$\begin{array}{c}\text { 处理 } \\
\text { Treatment }\end{array}$} & \multicolumn{6}{|c|}{ 栽后天数 Days after planting (d) } \\
\hline & & & & 50 & 70 & 90 & 110 & 130 & 150 \\
\hline \multirow[t]{24}{*}{2017} & 叶片 & 商薯 19 & SS & $3.22 \mathrm{a}$ & $4.27 \mathrm{a}$ & $4.39 \mathrm{a}$ & $5.86 \mathrm{a}$ & $6.74 \mathrm{a}$ & $5.11 \mathrm{a}$ \\
\hline & Leaf & Shangshu 19 & CK & $1.87 \mathrm{~b}$ & $2.96 \mathrm{~b}$ & $3.37 \mathrm{~b}$ & $5.22 \mathrm{~b}$ & $5.58 \mathrm{~b}$ & $4.76 \mathrm{~b}$ \\
\hline & & & JS & $1.53 \mathrm{c}$ & $2.74 \mathrm{~b}$ & $3.01 \mathrm{c}$ & $4.70 \mathrm{c}$ & $4.81 \mathrm{c}$ & $4.41 \mathrm{c}$ \\
\hline & & 济徐 23 & SS & $3.75 \mathrm{a}$ & $4.18 \mathrm{a}$ & $4.25 \mathrm{a}$ & $5.61 \mathrm{a}$ & $6.94 \mathrm{a}$ & $4.60 \mathrm{a}$ \\
\hline & & Jixu 23 & $\mathrm{CK}$ & $2.91 \mathrm{~b}$ & $3.33 \mathrm{~b}$ & $3.51 \mathrm{~b}$ & $4.59 \mathrm{~b}$ & $5.70 \mathrm{~b}$ & $4.19 \mathrm{~b}$ \\
\hline & & & JS & $2.04 \mathrm{c}$ & $2.85 \mathrm{c}$ & $3.36 \mathrm{c}$ & $4.31 \mathrm{c}$ & $4.95 \mathrm{c}$ & $4.02 \mathrm{~b}$ \\
\hline & 叶柄 & 商薯 19 & SS & $10.31 \mathrm{a}$ & $10.89 \mathrm{a}$ & $12.50 \mathrm{a}$ & $13.04 \mathrm{a}$ & $13.23 \mathrm{a}$ & $10.76 \mathrm{a}$ \\
\hline & Petiole & Shangshu 19 & CK & $6.80 \mathrm{~b}$ & $7.94 \mathrm{~b}$ & $7.97 \mathrm{~b}$ & $8.57 \mathrm{~b}$ & $8.69 \mathrm{~b}$ & $6.53 \mathrm{~b}$ \\
\hline & & & JS & $5.88 \mathrm{c}$ & $6.93 \mathrm{c}$ & $7.50 \mathrm{c}$ & $7.85 \mathrm{c}$ & $7.91 \mathrm{~b}$ & $5.78 \mathrm{c}$ \\
\hline & & 济徐 23 & SS & $10.24 \mathrm{a}$ & $10.61 \mathrm{a}$ & $10.35 \mathrm{a}$ & $11.56 \mathrm{a}$ & $12.55 \mathrm{a}$ & $10.30 \mathrm{a}$ \\
\hline & & Jixu 23 & CK & $7.48 \mathrm{~b}$ & $7.64 \mathrm{~b}$ & $7.93 \mathrm{~b}$ & $8.33 \mathrm{~b}$ & $8.55 \mathrm{~b}$ & $6.83 \mathrm{~b}$ \\
\hline & & & JS & $5.45 \mathrm{c}$ & $6.01 \mathrm{c}$ & $7.06 \mathrm{c}$ & $8.01 \mathrm{~b}$ & $8.34 \mathrm{~b}$ & $6.04 \mathrm{c}$ \\
\hline & 茎蔓 & 商薯 19 & SS & $3.82 \mathrm{c}$ & $5.37 \mathrm{c}$ & $7.01 \mathrm{c}$ & $7.31 \mathrm{c}$ & $7.82 \mathrm{c}$ & $5.77 \mathrm{c}$ \\
\hline & Stem & Shangshu 19 & $\mathrm{CK}$ & $6.48 \mathrm{~b}$ & $7.14 b$ & $8.26 \mathrm{~b}$ & $8.75 \mathrm{~b}$ & $9.47 \mathrm{~b}$ & $8.07 \mathrm{~b}$ \\
\hline & & & JS & $7.84 \mathrm{a}$ & $8.48 \mathrm{a}$ & $9.46 \mathrm{a}$ & $9.67 \mathrm{a}$ & $9.73 \mathrm{a}$ & $9.16 \mathrm{a}$ \\
\hline & & 济徐 23 & SS & $4.62 \mathrm{c}$ & $5.52 \mathrm{c}$ & $5.88 \mathrm{c}$ & $6.62 \mathrm{c}$ & $8.00 \mathrm{c}$ & $6.65 \mathrm{c}$ \\
\hline & & Jixu 23 & $\mathrm{CK}$ & $6.11 \mathrm{~b}$ & $6.57 \mathrm{~b}$ & $7.82 \mathrm{~b}$ & $8.91 \mathrm{~b}$ & $9.48 \mathrm{~b}$ & $9.21 \mathrm{~b}$ \\
\hline & & & JS & $7.32 \mathrm{a}$ & $8.01 \mathrm{a}$ & $9.21 \mathrm{a}$ & $9.89 \mathrm{a}$ & $10.20 \mathrm{a}$ & $9.98 \mathrm{a}$ \\
\hline & 块根 & 商薯 19 & SS & $7.40 \mathrm{a}$ & $7.74 \mathrm{a}$ & $8.31 \mathrm{a}$ & $9.10 \mathrm{a}$ & $11.26 \mathrm{a}$ & $8.92 \mathrm{a}$ \\
\hline & Storage root & Shangshu 19 & CK & $6.04 \mathrm{~b}$ & $6.19 \mathrm{~b}$ & $6.49 \mathrm{~b}$ & $6.70 \mathrm{~b}$ & $9.30 \mathrm{~b}$ & $6.95 \mathrm{~b}$ \\
\hline & & & JS & $5.50 \mathrm{c}$ & $6.01 \mathrm{c}$ & $5.81 \mathrm{c}$ & $6.33 \mathrm{c}$ & $8.96 \mathrm{c}$ & $6.42 \mathrm{c}$ \\
\hline & & 济徐 23 & SS & $7.35 \mathrm{a}$ & $8.62 \mathrm{a}$ & $9.31 \mathrm{a}$ & $9.26 \mathrm{a}$ & $10.82 \mathrm{a}$ & $9.20 \mathrm{a}$ \\
\hline & & Jixu 23 & CK & $5.70 \mathrm{~b}$ & $6.48 \mathrm{~b}$ & $7.41 \mathrm{~b}$ & $7.44 \mathrm{~b}$ & $9.08 \mathrm{~b}$ & $6.82 \mathrm{~b}$ \\
\hline & & & JS & $5.13 \mathrm{c}$ & $5.76 \mathrm{c}$ & $6.73 \mathrm{c}$ & $6.98 \mathrm{c}$ & $9.59 \mathrm{c}$ & $5.81 \mathrm{c}$ \\
\hline \multirow[t]{24}{*}{2018} & 叶片 & 商薯 19 & SS & $2.97 \mathrm{a}$ & $3.74 \mathrm{a}$ & $4.57 \mathrm{a}$ & $4.68 \mathrm{a}$ & $3.59 \mathrm{a}$ & $4.82 \mathrm{a}$ \\
\hline & Leaf & Shangshu 19 & CK & $1.85 \mathrm{~b}$ & $2.88 \mathrm{~b}$ & $3.64 \mathrm{~b}$ & $3.96 \mathrm{~b}$ & $4.74 \mathrm{~b}$ & $4.43 \mathrm{~b}$ \\
\hline & & & JS & $1.45 \mathrm{c}$ & $2.29 \mathrm{c}$ & $3.03 \mathrm{c}$ & $3.46 \mathrm{c}$ & $3.81 \mathrm{c}$ & $3.39 \mathrm{c}$ \\
\hline & & 济徐 23 & SS & $2.32 \mathrm{a}$ & $3.48 \mathrm{a}$ & $4.05 \mathrm{a}$ & $4.35 \mathrm{a}$ & $4.88 \mathrm{a}$ & $4.62 \mathrm{a}$ \\
\hline & & Jixu 23 & CK & $1.88 \mathrm{~b}$ & $2.80 \mathrm{~b}$ & $3.53 \mathrm{~b}$ & $3.69 \mathrm{~b}$ & $4.03 \mathrm{~b}$ & $3.54 \mathrm{~b}$ \\
\hline & & & JS & $1.08 \mathrm{c}$ & $2.43 \mathrm{c}$ & $2.87 \mathrm{c}$ & $3.17 \mathrm{c}$ & $3.57 \mathrm{c}$ & $3.54 \mathrm{~b}$ \\
\hline & 叶柄 & 商薯 19 & SS & $8.26 \mathrm{a}$ & $9.58 \mathrm{a}$ & $13.00 \mathrm{a}$ & $14.54 \mathrm{a}$ & $14.93 \mathrm{a}$ & $13.55 \mathrm{a}$ \\
\hline & Petiole & Shangshu 19 & CK & $6.80 \mathrm{~b}$ & $7.40 \mathrm{~b}$ & $9.03 \mathrm{~b}$ & $10.58 \mathrm{~b}$ & $11.89 \mathrm{~b}$ & $10.62 \mathrm{~b}$ \\
\hline & & & JS & $5.97 \mathrm{c}$ & $6.42 \mathrm{c}$ & $8.23 \mathrm{c}$ & $8.62 \mathrm{c}$ & $8.87 \mathrm{c}$ & $10.45 \mathrm{~b}$ \\
\hline & & 济徐 23 & SS & $9.10 \mathrm{a}$ & $9.94 \mathrm{a}$ & $14.07 \mathrm{a}$ & $14.25 \mathrm{a}$ & $15.25 \mathrm{a}$ & $12.44 \mathrm{a}$ \\
\hline & & Jixu 23 & CK & $7.29 \mathrm{~b}$ & $7.96 \mathrm{~b}$ & $9.83 \mathrm{~b}$ & $11.20 \mathrm{~b}$ & $10.89 \mathrm{~b}$ & $10.10 \mathrm{~b}$ \\
\hline & & & JS & $5.88 \mathrm{c}$ & $7.33 \mathrm{c}$ & $8.34 \mathrm{c}$ & $8.60 \mathrm{c}$ & $8.38 \mathrm{c}$ & $9.99 \mathrm{~b}$ \\
\hline & 茎蔓 & 商薯 19 & SS & $4.65 \mathrm{c}$ & $5.71 \mathrm{c}$ & $7.07 \mathrm{c}$ & $7.49 \mathrm{c}$ & $8.33 \mathrm{c}$ & $7.25 \mathrm{c}$ \\
\hline & Stem & Shangshu 19 & $\mathrm{CK}$ & $6.02 \mathrm{~b}$ & $7.75 \mathrm{~b}$ & $9.17 \mathrm{~b}$ & $9.27 \mathrm{~b}$ & $10.35 \mathrm{~b}$ & $9.10 \mathrm{~b}$ \\
\hline & & & JS & $7.11 \mathrm{a}$ & $9.17 \mathrm{a}$ & $10.42 \mathrm{a}$ & $10.74 \mathrm{a}$ & $11.22 \mathrm{a}$ & $10.52 \mathrm{a}$ \\
\hline & & 济徐 23 & SS & $4.98 \mathrm{c}$ & $6.00 \mathrm{c}$ & $6.76 \mathrm{c}$ & $7.18 \mathrm{c}$ & $8.34 \mathrm{c}$ & $7.06 \mathrm{c}$ \\
\hline & & Jixu 23 & CK & $6.61 \mathrm{~b}$ & $6.97 \mathrm{~b}$ & $8.84 \mathrm{~b}$ & $9.19 \mathrm{~b}$ & $10.29 \mathrm{~b}$ & $8.50 \mathrm{~b}$ \\
\hline & & & JS & $7.58 \mathrm{a}$ & $9.69 \mathrm{a}$ & $10.58 \mathrm{a}$ & $11.16 \mathrm{a}$ & $11.65 \mathrm{a}$ & $9.83 \mathrm{a}$ \\
\hline & 块根 & 商薯 19 & SS & $5.60 \mathrm{a}$ & $7.29 \mathrm{a}$ & $9.85 \mathrm{a}$ & $11.02 \mathrm{a}$ & $12.72 \mathrm{a}$ & $11.49 \mathrm{a}$ \\
\hline & Storage root & Shangshu 19 & CK & $4.28 \mathrm{~b}$ & $5.14 \mathrm{~b}$ & $6.03 \mathrm{~b}$ & $7.68 \mathrm{~b}$ & $8.36 \mathrm{~b}$ & $7.25 \mathrm{~b}$ \\
\hline & & & JS & $3.57 \mathrm{c}$ & $4.15 \mathrm{c}$ & $4.66 \mathrm{c}$ & $6.04 \mathrm{c}$ & $6.24 \mathrm{c}$ & $5.96 \mathrm{c}$ \\
\hline & & 济徐 23 & SS & $5.19 \mathrm{a}$ & $7.29 \mathrm{a}$ & $10.24 \mathrm{a}$ & $11.78 \mathrm{a}$ & $12.93 \mathrm{a}$ & $11.99 \mathrm{a}$ \\
\hline & & Jixu 23 & $\mathrm{CK}$ & $3.68 \mathrm{~b}$ & $4.99 \mathrm{~b}$ & $6.16 \mathrm{~b}$ & $7.05 \mathrm{~b}$ & $7.75 \mathrm{~b}$ & $7.00 \mathrm{~b}$ \\
\hline & & & JS & $3.20 \mathrm{c}$ & $4.13 \mathrm{c}$ & $5.14 \mathrm{c}$ & $5.85 \mathrm{c}$ & $6.49 \mathrm{c}$ & $5.42 \mathrm{c}$ \\
\hline
\end{tabular}

标以不同字母的值在处理间差异显著 $(P<0.05)$ 。缩写同表 1 。

Values followed by different letters within the same column are significantly different among different treatments at the 0.05 probability level Abbreviations are the same as those given in Table 1. 
表 5 不同器官淀粉含量

Table 5 Starch content in different organs (\%)

\begin{tabular}{|c|c|c|c|c|c|c|c|c|c|}
\hline \multirow{2}{*}{$\begin{array}{l}\text { 年份 } \\
\text { Year }\end{array}$} & \multirow{2}{*}{$\begin{array}{l}\text { 器官 } \\
\text { Organ }\end{array}$} & \multirow{2}{*}{$\begin{array}{c}\text { 品种 } \\
\text { Variety }\end{array}$} & \multirow{2}{*}{$\begin{array}{c}\text { 处理 } \\
\text { Treatment }\end{array}$} & \multicolumn{6}{|c|}{ 栽后天数 Days after planting (d) } \\
\hline & & & & 50 & 70 & 90 & 110 & 130 & 150 \\
\hline \multirow[t]{24}{*}{2017} & 叶片 & 商薯 19 & SS & $4.29 \mathrm{a}$ & $5.27 \mathrm{a}$ & $7.11 \mathrm{c}$ & $9.05 \mathrm{c}$ & $9.96 \mathrm{c}$ & $9.52 \mathrm{c}$ \\
\hline & Leaf & Shangshu 19 & CK & $3.64 \mathrm{~b}$ & $4.64 \mathrm{~b}$ & $8.96 \mathrm{~b}$ & $11.38 \mathrm{~b}$ & $11.60 \mathrm{~b}$ & $10.84 \mathrm{~b}$ \\
\hline & & & JS & $3.44 \mathrm{c}$ & $4.59 \mathrm{~b}$ & $9.59 \mathrm{a}$ & $12.87 \mathrm{a}$ & $14.28 \mathrm{a}$ & $12.07 \mathrm{a}$ \\
\hline & & 济徐 23 & SS & $5.48 \mathrm{a}$ & $6.32 \mathrm{a}$ & $7.37 \mathrm{~b}$ & $8.89 \mathrm{~b}$ & $10.16 \mathrm{c}$ & $8.87 \mathrm{c}$ \\
\hline & & Jixu 23 & $\mathrm{CK}$ & $4.63 \mathrm{~b}$ & $5.53 \mathrm{~b}$ & $9.94 \mathrm{a}$ & $11.99 \mathrm{a}$ & $12.21 \mathrm{~b}$ & $11.81 \mathrm{~b}$ \\
\hline & & & JS & $4.32 \mathrm{c}$ & $5.19 \mathrm{c}$ & $10.39 \mathrm{a}$ & $12.15 \mathrm{a}$ & $13.71 \mathrm{a}$ & $12.24 \mathrm{a}$ \\
\hline & 叶柄 & 商薯 19 & SS & $6.16 \mathrm{a}$ & $7.21 \mathrm{c}$ & $9.33 \mathrm{c}$ & $10.69 \mathrm{~b}$ & $10.86 \mathrm{c}$ & $8.17 \mathrm{c}$ \\
\hline & Petiole & Shangshu 19 & CK & $5.09 \mathrm{~b}$ & $7.94 \mathrm{~b}$ & $10.95 \mathrm{~b}$ & $12.14 \mathrm{a}$ & $12.50 \mathrm{~b}$ & $9.88 \mathrm{~b}$ \\
\hline & & & JS & $4.40 \mathrm{c}$ & $9.27 \mathrm{a}$ & $12.33 \mathrm{a}$ & $12.43 \mathrm{a}$ & $13.90 \mathrm{a}$ & $11.51 \mathrm{a}$ \\
\hline & & 济徐 23 & SS & $7.64 \mathrm{a}$ & $7.82 \mathrm{c}$ & $9.11 \mathrm{c}$ & $9.70 \mathrm{c}$ & $10.14 \mathrm{c}$ & $7.45 \mathrm{c}$ \\
\hline & & Jixu 23 & $\mathrm{CK}$ & $6.11 \mathrm{~b}$ & $8.45 \mathrm{~b}$ & $10.56 \mathrm{~b}$ & $11.09 \mathrm{~b}$ & $11.73 \mathrm{~b}$ & $9.36 \mathrm{~b}$ \\
\hline & & & JS & $5.58 \mathrm{c}$ & $9.69 \mathrm{a}$ & $12.15 \mathrm{a}$ & $12.38 \mathrm{a}$ & $13.81 \mathrm{a}$ & $11.13 \mathrm{a}$ \\
\hline & 茎蔓 & 商薯 19 & SS & $16.01 \mathrm{c}$ & $16.85 \mathrm{c}$ & $19.44 \mathrm{c}$ & $15.54 \mathrm{c}$ & $17.06 \mathrm{c}$ & $12.86 \mathrm{~b}$ \\
\hline & Stem & Shangshu 19 & $\mathrm{CK}$ & $18.37 \mathrm{~b}$ & $19.05 \mathrm{~b}$ & $21.61 \mathrm{~b}$ & $19.02 \mathrm{~b}$ & $19.75 \mathrm{~b}$ & $13.34 \mathrm{a}$ \\
\hline & & & JS & $19.81 \mathrm{a}$ & $20.71 \mathrm{a}$ & $22.68 \mathrm{a}$ & $20.20 \mathrm{a}$ & $21.80 \mathrm{a}$ & $13.39 \mathrm{a}$ \\
\hline & & 济徐 23 & SS & $15.45 \mathrm{c}$ & $16.32 \mathrm{c}$ & $19.08 \mathrm{c}$ & $16.32 \mathrm{c}$ & $17.15 \mathrm{c}$ & $13.81 \mathrm{c}$ \\
\hline & & Jixu 23 & $\mathrm{CK}$ & $18.81 \mathrm{~b}$ & $19.17 \mathrm{~b}$ & $21.62 \mathrm{~b}$ & $18.33 \mathrm{~b}$ & $18.79 \mathrm{~b}$ & $14.33 \mathrm{~b}$ \\
\hline & & & JS & $20.69 \mathrm{a}$ & $21.52 \mathrm{a}$ & $23.85 \mathrm{a}$ & $19.52 \mathrm{a}$ & $20.61 \mathrm{a}$ & $15.68 \mathrm{a}$ \\
\hline & 块根 & 商薯 19 & SS & $38.74 \mathrm{a}$ & $40.78 \mathrm{a}$ & $61.29 \mathrm{a}$ & $67.69 \mathrm{a}$ & $79.46 \mathrm{a}$ & $88.66 \mathrm{a}$ \\
\hline & Storage root & Shangshu 19 & CK & $24.75 \mathrm{~b}$ & $29.61 \mathrm{~b}$ & $44.92 \mathrm{~b}$ & $58.35 \mathrm{~b}$ & $66.81 \mathrm{~b}$ & $71.28 \mathrm{~b}$ \\
\hline & & & JS & $22.24 \mathrm{c}$ & $25.92 \mathrm{c}$ & $38.34 \mathrm{c}$ & $51.25 \mathrm{c}$ & $61.07 \mathrm{c}$ & $64.95 \mathrm{c}$ \\
\hline & & 济徐 23 & SS & $35.49 \mathrm{a}$ & $38.6 \mathrm{a}$ & $58.22 \mathrm{a}$ & $68.65 \mathrm{a}$ & $81.63 \mathrm{a}$ & $87.81 \mathrm{a}$ \\
\hline & & Jixu 23 & $\mathrm{CK}$ & $26.98 \mathrm{~b}$ & $30.86 \mathrm{~b}$ & $45.86 \mathrm{~b}$ & $59.61 \mathrm{~b}$ & $65.61 \mathrm{~b}$ & $69.75 \mathrm{~b}$ \\
\hline & & & JS & $24.31 \mathrm{c}$ & $27.9 \mathrm{c}$ & $38.79 \mathrm{c}$ & $51.04 \mathrm{c}$ & $58.05 \mathrm{c}$ & $60.52 \mathrm{c}$ \\
\hline \multirow[t]{24}{*}{2018} & 叶片 & 商薯 19 & SS & $2.21 \mathrm{a}$ & $3.83 \mathrm{a}$ & $5.61 \mathrm{a}$ & $6.39 \mathrm{c}$ & $8.05 \mathrm{c}$ & $8.25 \mathrm{c}$ \\
\hline & Leaf & Shangshu 19 & CK & $1.72 \mathrm{~b}$ & $3.04 \mathrm{~b}$ & $5.62 \mathrm{a}$ & $7.25 \mathrm{~b}$ & $10.16 \mathrm{~b}$ & $9.88 \mathrm{~b}$ \\
\hline & & & JS & $1.52 \mathrm{c}$ & $2.69 \mathrm{c}$ & $5.63 \mathrm{a}$ & $8.33 \mathrm{a}$ & $11.71 \mathrm{a}$ & $10.47 \mathrm{a}$ \\
\hline & & 济徐 23 & SS & $2.02 \mathrm{a}$ & $4.06 \mathrm{a}$ & $5.11 \mathrm{a}$ & $5.92 \mathrm{c}$ & $8.11 \mathrm{c}$ & $7.82 \mathrm{c}$ \\
\hline & & Jixu 23 & CK & $1.72 \mathrm{~b}$ & $3.31 \mathrm{~b}$ & $5.27 \mathrm{a}$ & $7.45 \mathrm{~b}$ & $9.60 \mathrm{~b}$ & $8.28 \mathrm{~b}$ \\
\hline & & & JS & $1.44 \mathrm{c}$ & $2.32 \mathrm{c}$ & $5.38 \mathrm{a}$ & $8.30 \mathrm{a}$ & $11.17 \mathrm{c}$ & $10.19 \mathrm{a}$ \\
\hline & 叶柄 & 商薯 19 & SS & $5.06 \mathrm{a}$ & $6.70 \mathrm{a}$ & $7.70 \mathrm{c}$ & $10.97 \mathrm{c}$ & $11.56 \mathrm{c}$ & $10.22 \mathrm{c}$ \\
\hline & Petiole & Shangshu 19 & CK & $4.33 \mathrm{~b}$ & $6.52 \mathrm{ab}$ & $8.70 \mathrm{~b}$ & $13.42 \mathrm{~b}$ & $14.64 \mathrm{~b}$ & $12.78 \mathrm{~b}$ \\
\hline & & & JS & $3.74 \mathrm{c}$ & $6.20 \mathrm{~b}$ & $10.07 \mathrm{a}$ & $15.33 \mathrm{a}$ & $16.02 \mathrm{a}$ & $14.43 \mathrm{a}$ \\
\hline & & 济徐 23 & SS & $5.79 \mathrm{a}$ & $6.57 \mathrm{a}$ & $8.26 \mathrm{c}$ & $11.46 \mathrm{c}$ & $11.23 \mathrm{c}$ & $10.25 \mathrm{c}$ \\
\hline & & Jixu 23 & $\mathrm{CK}$ & $3.96 \mathrm{~b}$ & $6.47 \mathrm{a}$ & $10.21 \mathrm{~b}$ & $14.00 \mathrm{~b}$ & $15.29 \mathrm{~b}$ & $12.41 \mathrm{~b}$ \\
\hline & & & JS & $3.40 \mathrm{c}$ & $6.43 \mathrm{a}$ & $11.32 \mathrm{c}$ & $15.19 \mathrm{a}$ & $16.91 \mathrm{a}$ & $14.59 \mathrm{a}$ \\
\hline & 茎蔓 & 商薯 19 & SS & $12.85 \mathrm{c}$ & $13.90 \mathrm{c}$ & $14.98 \mathrm{c}$ & $15.66 \mathrm{c}$ & $16.36 \mathrm{c}$ & $15.13 \mathrm{c}$ \\
\hline & Stem & Shangshu 19 & CK & $14.13 \mathrm{~b}$ & $15.34 \mathrm{~b}$ & $17.60 \mathrm{~b}$ & $18.34 \mathrm{~b}$ & $19.42 \mathrm{~b}$ & $16.90 \mathrm{~b}$ \\
\hline & & & JS & $15.23 \mathrm{a}$ & $17.22 \mathrm{a}$ & $19.09 \mathrm{a}$ & $20.34 \mathrm{a}$ & $22.25 \mathrm{a}$ & $18.44 \mathrm{a}$ \\
\hline & & 济徐 23 & SS & $11.74 \mathrm{c}$ & $12.41 \mathrm{c}$ & $13.87 \mathrm{c}$ & $14.86 \mathrm{c}$ & $15.46 \mathrm{c}$ & $12.48 \mathrm{c}$ \\
\hline & & Jixu 23 & $\mathrm{CK}$ & $13.23 \mathrm{~b}$ & $14.74 \mathrm{~b}$ & $15.30 \mathrm{~b}$ & $16.98 \mathrm{~b}$ & $17.45 \mathrm{~b}$ & $13.92 \mathrm{~b}$ \\
\hline & & & JS & $14.92 \mathrm{a}$ & $16.70 \mathrm{a}$ & $18.78 \mathrm{a}$ & $21.23 \mathrm{a}$ & $22.68 \mathrm{a}$ & $17.69 \mathrm{a}$ \\
\hline & 块根 & 商薯 19 & SS & $34.04 \mathrm{a}$ & $41.80 \mathrm{a}$ & $60.46 \mathrm{a}$ & $66.33 \mathrm{a}$ & $76.01 \mathrm{a}$ & $83.07 \mathrm{a}$ \\
\hline & Storage root & Shangshu 19 & $\mathrm{CK}$ & $23.52 \mathrm{~b}$ & $32.00 \mathrm{~b}$ & $45.61 \mathrm{~b}$ & $47.44 \mathrm{~b}$ & $58.76 \mathrm{~b}$ & $70.06 \mathrm{~b}$ \\
\hline & & & JS & $20.72 \mathrm{c}$ & $27.10 \mathrm{c}$ & $40.10 \mathrm{c}$ & $41.89 \mathrm{c}$ & $50.12 \mathrm{c}$ & $60.51 \mathrm{c}$ \\
\hline & & 济徐 23 & SS & $37.92 \mathrm{a}$ & $44.20 \mathrm{a}$ & $62.13 \mathrm{a}$ & $65.75 \mathrm{a}$ & $75.70 \mathrm{a}$ & $86.02 \mathrm{a}$ \\
\hline & & Jixu 23 & $\mathrm{CK}$ & $22.92 \mathrm{~b}$ & $28.36 \mathrm{~b}$ & $42.12 \mathrm{~b}$ & $47.90 \mathrm{~b}$ & $58.03 \mathrm{~b}$ & $65.40 \mathrm{~b}$ \\
\hline & & & JS & $19.68 \mathrm{c}$ & $25.07 \mathrm{c}$ & $34.60 \mathrm{c}$ & $41.35 \mathrm{c}$ & $50.74 \mathrm{c}$ & $56.93 \mathrm{c}$ \\
\hline
\end{tabular}




\section{4 功能叶以下茎不同部位蔗糖和淀粉含量}

由表 6 可知, 茎基部蔗糖含量显著高于茎顶部和茎中 部。与对照处理相比, 疏松处理显著提高茎顶部蔗糖含量 而显著降低茎中部和基部蔗糖含量; 紧实处理变化规律
与之相反。疏松处理主茎各部位淀粉含量均显著下降, 而 紧实处理主茎各部位淀粉含量均显著提高, 2 个处理均以 茎中部和基部的变化幅度较大。即改善土壤通气性减少了 蔗糖和淀粉在茎中部和基部的积累。

表 6 茎不同部位蔗糖和淀粉含量 $(\%, 2018$ 年 $)$

Table 6 Contents of sucrose and starch in different positions of stem $(\%, 2018)$

\begin{tabular}{|c|c|c|c|c|c|c|c|c|c|}
\hline \multirow{2}{*}{$\begin{array}{l}\text { 项目 } \\
\text { Item }\end{array}$} & \multirow{2}{*}{$\begin{array}{c}\text { 部位 } \\
\text { Position }\end{array}$} & \multirow{2}{*}{$\begin{array}{c}\text { 品种 } \\
\text { Variety }\end{array}$} & \multirow{2}{*}{$\begin{array}{c}\text { 处理 } \\
\text { Treatment }\end{array}$} & \multicolumn{6}{|c|}{ 栽后天数 Days after planting (d) } \\
\hline & & & & 50 & 70 & 90 & 110 & 130 & 150 \\
\hline \multirow{18}{*}{$\begin{array}{c}\text { 蔗糖 } \\
\text { Sucrose }\end{array}$} & \multirow{6}{*}{$\begin{array}{c}\text { 茎顶部 } \\
\text { Stem top }\end{array}$} & 商薯 19 & SS & $3.07 \mathrm{a}$ & $4.45 \mathrm{a}$ & $5.03 \mathrm{a}$ & $5.36 \mathrm{a}$ & $5.87 \mathrm{a}$ & $5.40 \mathrm{a}$ \\
\hline & & Shangshu 19 & CK & $1.64 \mathrm{~b}$ & $3.25 \mathrm{~b}$ & $4.33 \mathrm{~b}$ & $4.74 \mathrm{~b}$ & $5.37 \mathrm{~b}$ & $4.88 \mathrm{~b}$ \\
\hline & & & JS & $1.19 \mathrm{c}$ & $2.46 \mathrm{c}$ & $3.85 \mathrm{c}$ & $4.25 \mathrm{c}$ & $4.71 \mathrm{c}$ & $4.48 \mathrm{c}$ \\
\hline & & 济徐 23 & SS & $2.50 \mathrm{a}$ & $4.61 \mathrm{a}$ & $5.13 \mathrm{a}$ & $5.72 \mathrm{a}$ & $6.25 \mathrm{a}$ & $5.26 \mathrm{a}$ \\
\hline & & Jixu 23 & CK & $2.07 \mathrm{~b}$ & $3.18 \mathrm{~b}$ & $4.47 \mathrm{~b}$ & $5.10 \mathrm{~b}$ & $5.43 \mathrm{~b}$ & $4.36 \mathrm{~b}$ \\
\hline & & & JS & $1.70 \mathrm{c}$ & $2.49 \mathrm{c}$ & $3.91 \mathrm{c}$ & $4.73 \mathrm{c}$ & $4.74 \mathrm{c}$ & $4.00 \mathrm{c}$ \\
\hline & \multirow{6}{*}{$\begin{array}{c}\text { 茎中部 } \\
\text { Stem middle }\end{array}$} & 商薯 19 & SS & $2.60 \mathrm{c}$ & $2.87 \mathrm{c}$ & $3.35 \mathrm{c}$ & $4.07 \mathrm{c}$ & $5.58 \mathrm{c}$ & $4.44 \mathrm{c}$ \\
\hline & & Shangshu 19 & CK & $3.76 \mathrm{~b}$ & $3.24 \mathrm{~b}$ & $4.12 \mathrm{~b}$ & $5.30 \mathrm{~b}$ & $6.60 \mathrm{~b}$ & $5.82 \mathrm{~b}$ \\
\hline & & & JS & $5.13 \mathrm{a}$ & $4.37 \mathrm{a}$ & $5.69 \mathrm{a}$ & $6.71 \mathrm{a}$ & $8.24 \mathrm{a}$ & $6.75 \mathrm{a}$ \\
\hline & & 济徐 23 & SS & $1.69 \mathrm{c}$ & $2.48 \mathrm{c}$ & $3.33 \mathrm{c}$ & $4.12 \mathrm{c}$ & $5.45 \mathrm{c}$ & $3.93 \mathrm{c}$ \\
\hline & & Jixu 23 & CK & $2.08 \mathrm{~b}$ & $3.14 \mathrm{~b}$ & $4.01 \mathrm{~b}$ & $5.32 \mathrm{~b}$ & $6.26 \mathrm{~b}$ & $4.97 \mathrm{~b}$ \\
\hline & & & JS & $4.71 \mathrm{a}$ & $4.54 \mathrm{a}$ & $5.33 \mathrm{a}$ & $7.01 \mathrm{a}$ & $7.96 \mathrm{a}$ & $6.25 \mathrm{a}$ \\
\hline & \multirow{6}{*}{$\begin{array}{c}\text { 茎基部 } \\
\text { Stem base }\end{array}$} & 商薯 19 & SS & $8.75 \mathrm{c}$ & $9.58 \mathrm{c}$ & $10.10 \mathrm{c}$ & $10.41 \mathrm{c}$ & $11.34 \mathrm{c}$ & $10.27 \mathrm{c}$ \\
\hline & & Shangshu 19 & $\mathrm{CK}$ & $9.87 \mathrm{~b}$ & $11.50 \mathrm{~b}$ & $12.84 \mathrm{~b}$ & $11.69 \mathrm{~b}$ & $12.51 \mathrm{~b}$ & $11.51 \mathrm{~b}$ \\
\hline & & & JS & $10.91 \mathrm{a}$ & $13.32 \mathrm{a}$ & $14.88 \mathrm{a}$ & $14.47 \mathrm{a}$ & $15.47 \mathrm{a}$ & $13.79 \mathrm{a}$ \\
\hline & & 济徐 23 & SS & $8.30 \mathrm{c}$ & $9.53 \mathrm{c}$ & $9.93 \mathrm{c}$ & $9.94 \mathrm{c}$ & $10.89 \mathrm{c}$ & $9.52 \mathrm{c}$ \\
\hline & & Jixu 23 & CK & $9.93 \mathrm{~b}$ & $11.20 \mathrm{~b}$ & $12.10 \mathrm{~b}$ & $11.33 \mathrm{~b}$ & $12.36 \mathrm{~b}$ & $11.12 \mathrm{~b}$ \\
\hline & & & JS & $11.40 \mathrm{a}$ & $13.41 \mathrm{a}$ & $14.49 \mathrm{a}$ & $14.27 \mathrm{a}$ & $14.86 \mathrm{a}$ & $13.35 \mathrm{a}$ \\
\hline \multirow{18}{*}{$\begin{array}{l}\text { 淀粉 } \\
\text { Starch }\end{array}$} & \multirow{6}{*}{$\begin{array}{l}\text { 茎顶部 } \\
\text { Stem top }\end{array}$} & 商薯 19 & SS & $11.93 \mathrm{c}$ & $13.12 \mathrm{c}$ & $14.53 \mathrm{c}$ & $15.40 \mathrm{c}$ & $16.17 \mathrm{c}$ & $14.46 \mathrm{c}$ \\
\hline & & Shangshu 19 & $\mathrm{CK}$ & $13.17 \mathrm{~b}$ & $14.49 \mathrm{~b}$ & $16.82 \mathrm{~b}$ & $17.79 \mathrm{~b}$ & $18.17 \mathrm{~b}$ & $16.50 \mathrm{~b}$ \\
\hline & & & JS & $14.53 \mathrm{a}$ & $16.36 \mathrm{a}$ & $18.79 \mathrm{a}$ & $20.09 \mathrm{a}$ & $21.11 \mathrm{a}$ & $18.12 \mathrm{a}$ \\
\hline & & 济徐 23 & SS & $11.99 \mathrm{c}$ & $12.26 \mathrm{c}$ & $13.24 \mathrm{c}$ & $15.02 \mathrm{c}$ & $15.58 \mathrm{c}$ & $11.86 \mathrm{c}$ \\
\hline & & Jixu 23 & CK & $13.07 \mathrm{~b}$ & $14.66 \mathrm{~b}$ & $15.30 \mathrm{~b}$ & $16.30 \mathrm{~b}$ & $17.41 \mathrm{~b}$ & $13.19 \mathrm{~b}$ \\
\hline & & & JS & $14.39 \mathrm{a}$ & $16.54 \mathrm{a}$ & $18.36 \mathrm{a}$ & $19.53 \mathrm{a}$ & $20.47 \mathrm{a}$ & $16.65 \mathrm{a}$ \\
\hline & \multirow{6}{*}{$\begin{array}{c}\text { 茎中部 } \\
\text { Stem middle }\end{array}$} & 商薯 19 & SS & $14.11 \mathrm{c}$ & $14.89 \mathrm{c}$ & $15.72 \mathrm{c}$ & $17.17 \mathrm{c}$ & $18.19 \mathrm{c}$ & $17.09 \mathrm{c}$ \\
\hline & & Shangshu 19 & $\mathrm{CK}$ & $15.37 \mathrm{~b}$ & $17.59 \mathrm{~b}$ & $17.89 \mathrm{~b}$ & $19.56 \mathrm{~b}$ & $20.47 \mathrm{~b}$ & $19.96 \mathrm{~b}$ \\
\hline & & & JS & $17.62 \mathrm{a}$ & $19.00 \mathrm{a}$ & $21.05 \mathrm{a}$ & $21.96 \mathrm{a}$ & $22.71 \mathrm{a}$ & $21.56 \mathrm{a}$ \\
\hline & & 济徐 23 & SS & $13.88 \mathrm{c}$ & $14.56 \mathrm{c}$ & $15.29 \mathrm{c}$ & $16.38 \mathrm{c}$ & $17.31 \mathrm{c}$ & $15.35 \mathrm{c}$ \\
\hline & & Jixu 23 & CK & $16.21 \mathrm{~b}$ & $18.18 \mathrm{~b}$ & $19.01 \mathrm{~b}$ & $19.62 \mathrm{~b}$ & $20.54 \mathrm{~b}$ & $17.76 \mathrm{~b}$ \\
\hline & & & JS & $18.28 \mathrm{a}$ & $20.07 \mathrm{a}$ & $20.85 \mathrm{a}$ & $22.27 \mathrm{a}$ & $22.52 \mathrm{a}$ & $20.05 \mathrm{a}$ \\
\hline & \multirow{6}{*}{$\begin{array}{c}\text { 茎基部 } \\
\text { Stem base }\end{array}$} & 商薯 19 & SS & $18.73 \mathrm{c}$ & $19.73 \mathrm{c}$ & $21.16 \mathrm{c}$ & $21.69 \mathrm{c}$ & $22.23 \mathrm{c}$ & $21.17 \mathrm{c}$ \\
\hline & & Shangshu 19 & CK & 22.32 & $23.34 \mathrm{c}$ & $24.36 \mathrm{c}$ & $24.29 \mathrm{c}$ & $25.49 \mathrm{c}$ & $24.62 \mathrm{~b}$ \\
\hline & & & JS & $24.69 \mathrm{a}$ & $25.57 \mathrm{a}$ & $26.47 \mathrm{a}$ & $27.40 \mathrm{a}$ & $28.84 \mathrm{a}$ & $27.85 \mathrm{a}$ \\
\hline & & 济徐 23 & SS & $17.56 \mathrm{c}$ & $19.59 \mathrm{c}$ & $50.80 \mathrm{c}$ & $21.22 \mathrm{c}$ & $21.92 \mathrm{c}$ & $20.17 \mathrm{c}$ \\
\hline & & Jixu 23 & $\mathrm{CK}$ & $21.34 \mathrm{~b}$ & $23.16 \mathrm{~b}$ & $23.88 \mathrm{~b}$ & $23.80 \mathrm{~b}$ & $25.11 \mathrm{~b}$ & $23.26 \mathrm{~b}$ \\
\hline & & & JS & $23.47 \mathrm{a}$ & $25.11 \mathrm{a}$ & $26.83 \mathrm{a}$ & $27.75 \mathrm{a}$ & $29.47 \mathrm{a}$ & $28.09 \mathrm{a}$ \\
\hline
\end{tabular}




\section{5 器官间蔗糖含量差}

器官间蔗糖含量差可以反映器官间光合产物运转的 情况。由表 7 可知, 疏松处理显著降低茎基部与茎顶部和 茎基部与块根间的蔗糖含量差, 而显著提高柄与叶片和 柄与茎顶部间的蔗糖含量差; 紧实处理显著提高茎基部
与茎顶部和茎基部与块根间的蔗糖含量差, 对柄与叶片 和柄与上茎的蔗糖含量差的影响存在年份差异。两处理引 起器官间蔗糖含量差的变化幅度表现为茎基部与块根间 $>$ 茎基部与茎顶部间 $>$ 柄与叶片间和柄与茎顶间。即改善土壤 通气性主要提高了茎基部到块根间光合产物的运转效率。

\section{表 7 不同器官间蔗糖含量差}

Table 7 Difference of sucrose contents between adjacent organs (\%)

\begin{tabular}{|c|c|c|c|c|c|c|c|}
\hline $\begin{array}{l}\text { 年份 } \\
\text { Year }\end{array}$ & $\begin{array}{c}\text { 栽后天数 } \\
\text { Days after } \\
\text { planting (d) }\end{array}$ & $\begin{array}{c}\text { 品种 } \\
\text { Variety }\end{array}$ & $\begin{array}{c}\text { 处理 } \\
\text { Treatment }\end{array}$ & $\begin{array}{c}\text { 柄-叶 } \\
\text { Petiole- } \\
\text { Leaf }\end{array}$ & $\begin{array}{c}\text { 柄-茎顶部 } \\
\text { Petiole- } \\
\text { Stem top } \\
\end{array}$ & $\begin{array}{c}\text { 茎基部-茎顶部 } \\
\text { Stem base- } \\
\text { Stem top } \\
\end{array}$ & $\begin{array}{c}\text { 茎基部-块根 } \\
\text { Stem top- } \\
\text { Storage root } \\
\end{array}$ \\
\hline \multirow[t]{18}{*}{2017} & 90 & 商薯 19 & SS & $96.03 \mathrm{a}$ & $55.62 \mathrm{a}$ & $10.34 \mathrm{c}$ & $-5.95 \mathrm{c}$ \\
\hline & & Shangshu 19 & CK & $81.13 \mathrm{~b}$ & $15.55 \mathrm{c}$ & $31.08 \mathrm{~b}$ & $35.90 \mathrm{~b}$ \\
\hline & & & JS & $85.44 \mathrm{~b}$ & $25.39 \mathrm{~b}$ & $60.84 \mathrm{a}$ & $60.84 \mathrm{a}$ \\
\hline & & 济徐 23 & SS & $83.56 \mathrm{a}$ & $35.90 \mathrm{a}$ & $-2.53 \mathrm{c}$ & $-28.05 \mathrm{c}$ \\
\hline & & Jixu 23 & CK & $77.27 \mathrm{~b}$ & $22.44 \mathrm{~b}$ & $44.57 \mathrm{~b}$ & $29.36 \mathrm{~b}$ \\
\hline & & & JS & $71.02 \mathrm{c}$ & $26.28 \mathrm{~b}$ & $63.22 \mathrm{a}$ & $43.12 \mathrm{a}$ \\
\hline & 110 & 商薯 19 & SS & $75.98 \mathrm{a}$ & $54.81 \mathrm{a}$ & $-1.36 \mathrm{c}$ & $-21.55 \mathrm{c}$ \\
\hline & & Shangshu 19 & $\mathrm{CK}$ & $48.59 \mathrm{~b}$ & $28.38 \mathrm{~b}$ & $30.64 \mathrm{~b}$ & $26.76 \mathrm{~b}$ \\
\hline & & & JS & $50.20 \mathrm{~b}$ & $32.25 \mathrm{~b}$ & $54.62 \mathrm{a}$ & $44.28 \mathrm{a}$ \\
\hline & & 济徐 23 & SS & $69.31 \mathrm{a}$ & $34.60 \mathrm{a}$ & $-22.53 c$ & $-35.03 \mathrm{c}$ \\
\hline & & Jixu 23 & $\mathrm{CK}$ & $57.89 \mathrm{~b}$ & $10.35 \mathrm{c}$ & $15.48 \mathrm{~b}$ & $16.41 \mathrm{~b}$ \\
\hline & & & JS & $60.06 \mathrm{~b}$ & $17.07 \mathrm{~b}$ & $45.18 \mathrm{a}$ & $41.99 \mathrm{a}$ \\
\hline & 130 & 商薯 19 & SS & $65.00 \mathrm{a}$ & $41.98 \mathrm{a}$ & $-36.07 \mathrm{c}$ & $-60.95 \mathrm{c}$ \\
\hline & & Shangshu 19 & CK & $43.59 \mathrm{c}$ & $22.68 \mathrm{c}$ & $25.80 \mathrm{~b}$ & $-3.61 \mathrm{~b}$ \\
\hline & & & JS & $48.74 \mathrm{~b}$ & $27.46 \mathrm{~b}$ & $44.76 \mathrm{a}$ & $5.43 \mathrm{a}$ \\
\hline & & 济徐 23 & SS & $57.57 \mathrm{a}$ & $33.05 \mathrm{a}$ & $-49.03 c$ & $-66.01 \mathrm{c}$ \\
\hline & & Jixu 23 & CK & $40.00 \mathrm{c}$ & $19.94 \mathrm{c}$ & $18.65 \mathrm{~b}$ & $-7.31 \mathrm{~b}$ \\
\hline & & & JS & $51.02 \mathrm{~b}$ & $29.75 \mathrm{~b}$ & $38.01 \mathrm{a}$ & $-5.46 \mathrm{a}$ \\
\hline \multirow[t]{18}{*}{2018} & 90 & 商薯 19 & SS & $95.99 \mathrm{a}$ & $88.36 \mathrm{a}$ & $67.04 \mathrm{c}$ & $2.56 \mathrm{c}$ \\
\hline & & Shangshu 19 & $\mathrm{CK}$ & $84.95 \mathrm{a}$ & $70.22 \mathrm{~b}$ & $99.07 \mathrm{~b}$ & $72.14 \mathrm{~b}$ \\
\hline & & & JS & $92.21 \mathrm{~b}$ & $72.52 \mathrm{~b}$ & $117.78 \mathrm{a}$ & $104.62 \mathrm{a}$ \\
\hline & & 济徐 23 & SS & $110.58 \mathrm{a}$ & $93.16 \mathrm{a}$ & $63.79 \mathrm{c}$ & $-3.02 \mathrm{c}$ \\
\hline & & Jixu 23 & $\mathrm{CK}$ & $94.39 \mathrm{c}$ & $75.00 \mathrm{~b}$ & $92.15 \mathrm{~b}$ & $65.08 \mathrm{~b}$ \\
\hline & & & JS & $97.68 \mathrm{~b}$ & $72.29 \mathrm{~b}$ & $114.98 \mathrm{a}$ & $95.33 \mathrm{a}$ \\
\hline & 110 & 商薯 19 & SS & $102.48 \mathrm{a}$ & $92.27 \mathrm{a}$ & $64.18 \mathrm{c}$ & $-5.68 \mathrm{c}$ \\
\hline & & Shangshu 19 & $\mathrm{CK}$ & $91.05 \mathrm{~b}$ & $76.23 \mathrm{~b}$ & $84.58 \mathrm{~b}$ & $41.45 \mathrm{~b}$ \\
\hline & & & JS & $85.38 \mathrm{~b}$ & $68.04 \mathrm{c}$ & $109.26 \mathrm{a}$ & $82.20 \mathrm{a}$ \\
\hline & & 济徐 23 & SS & $106.44 \mathrm{a}$ & $85.44 \mathrm{a}$ & $53.97 \mathrm{c}$ & $-16.85 \mathrm{c}$ \\
\hline & & Jixu 23 & $\mathrm{CK}$ & $100.85 \mathrm{~b}$ & $74.91 \mathrm{~b}$ & $75.91 \mathrm{~b}$ & $46.60 \mathrm{~b}$ \\
\hline & & & JS & $92.25 \mathrm{c}$ & $58.05 \mathrm{c}$ & $100.38 \mathrm{a}$ & $83.64 \mathrm{a}$ \\
\hline & 130 & 商薯 19 & SS & $91.07 \mathrm{a}$ & $87.05 \mathrm{a}$ & $63.50 \mathrm{c}$ & $-11.47 \mathrm{c}$ \\
\hline & & Shangshu 19 & CK & $85.95 \mathrm{~b}$ & $75.53 \mathrm{~b}$ & $79.91 \mathrm{~b}$ & $39.79 \mathrm{~b}$ \\
\hline & & & JS & $79.78 \mathrm{c}$ & $61.30 \mathrm{c}$ & $106.66 \mathrm{a}$ & $84.97 \mathrm{a}$ \\
\hline & & 济徐 23 & SS & $103.02 \mathrm{a}$ & $83.76 \mathrm{a}$ & $54.22 \mathrm{c}$ & $-17.08 \mathrm{c}$ \\
\hline & & Jixu 23 & $\mathrm{CK}$ & $92.04 \mathrm{~b}$ & $67.01 \mathrm{~b}$ & $77.99 \mathrm{~b}$ & $45.90 \mathrm{~b}$ \\
\hline & & & JS & $80.44 \mathrm{c}$ & $55.32 \mathrm{c}$ & $103.25 \mathrm{a}$ & $78.36 \mathrm{a}$ \\
\hline
\end{tabular}


2.6 甘薯器官间蔗糖含量差与块根碳水化合物含量的相 关分析

由表 8 可知, 茎基部与茎顶部和茎基部与块根间的蔗糖
含量差与块根中蔗糖和淀粉含量均呈极显著负相关, 且茎 基部与块根间蔗糖含量差与块根蔗糖含量相关系数较大、茎 基部与茎顶部间蔗糖含量差与块根淀粉含量相关系数较大。

表 8 甘薯器官间蔗糖含量差与块根碳水化合物含量的相关性分析

Table 8 Analysis of correlation coefficient between differences in sucrose content in sweet potato organs and sucrose and starch contents in tuber root

\begin{tabular}{|c|c|c|c|}
\hline $\begin{array}{c}\text { 品种 } \\
\text { Variety }\end{array}$ & $\begin{array}{c}\text { 相邻器官间蔗糖含量差 } \\
\text { Sucrose content differences between } \\
\text { adjacent organ }\end{array}$ & $\begin{array}{c}\text { 与块根蔗糖含量的相关系数 } \\
\text { Correlation coefficient of sucrose } \\
\text { content between adjacent organs with } \\
\text { sucrose content in storage root } \\
\end{array}$ & $\begin{array}{c}\text { 与块根淀粉含量的相关系数 } \\
\text { Correlation coefficient of sucrose content } \\
\text { between adjacent organs with starch content } \\
\text { in storage root }\end{array}$ \\
\hline \multirow{4}{*}{$\begin{array}{l}\text { 商薯 } 19 \\
\text { Shangshu } \\
19\end{array}$} & 柄-叶 Petiole-Leaf & -0.274 & $-0.679^{* *}$ \\
\hline & 柄-茎顶部 Petiole-Stem top & -0.308 & $-0.732^{* *}$ \\
\hline & 茎基部-茎顶部 Stem base-Stem top & $-0.758^{* *}$ & $-0.934^{* *}$ \\
\hline & 茎基部-块根 Stem top-Storage roots & $-0.924^{* *}$ & $-0.882^{* *}$ \\
\hline \multirow{4}{*}{$\begin{array}{c}\text { 济徐 } 23 \\
\text { Jixu } 23\end{array}$} & 柄-叶 Petiole-Leaf & -0.210 & $-0.582^{*}$ \\
\hline & 柄-茎顶部 Petiole-Stem top & -0.243 & $-0.651^{* *}$ \\
\hline & 茎基部-茎顶部 Stem base-Stem top & $-0.839^{* *}$ & $-0.933^{* *}$ \\
\hline & 茎基部-块根 Stem top-Storage roots & $-0.913^{* *}$ & $-0.856^{* *}$ \\
\hline
\end{tabular}

"表示在 0.05 水平(双侧)上显著相关, ${ }^{* *}$ 表示在 0.01 水平(双侧)上显著相关

"and ${ }^{* *}$ mean significant at the 0.05 and 0.01 probability levels, respectively.

\section{3 讨论}

\section{1 土壤通气性影响甘薯块根产量的原因分析}

改善土壤通气性能显著提高甘薯块根产量 ${ }^{[13,17,20-22]}$, 增加土壤容重或向土壤中冲入氮气，降低氧气浓度, 则显 著降低块根产量 ${ }^{[14-15]}$ 。土壤通气性好，块根形成早、数量 多, 块根产量高, 而土壤通气性差, 块根膨大慢 ${ }^{[14-15]}$, 块 根产量低。也有研究认为, 改善土壤通气性通过提高甘薯 单薯重和收获指数增加块根产量 ${ }^{[22]}$ 。本研究结果表明, 与 对照处理相比, 疏松处理能显著提高块根产量, 商薯 19 和济 徐 23 两年的平均增幅分别为 $27.03 \%$ 和 $38.74 \%$; 紧实处理 则显著降低两品种块根产量，降幅分别为 $17.87 \%$ 和 $15.92 \%$ (表 1)。与对照处理相比，疏松处理显著提高块根 中 ${ }^{13} \mathrm{C}$ 同化物的分配率(表 3), 块根单薯重和经济系数; 紧 实处理则提高地上部器官中 ${ }^{13} \mathrm{C}$ 同化物的分配率, 降低块 根单薯重和经济系数。即改善土壤通气性能通过促进光合 产物向块根中运转，促进块根膨大而增加块根产量。

3.2 土壤通气性引起甘薯源库间光合产物运转差异的原 因解析

目前，改善土壤通气性提高甘薯块根产量的原因分 析多集中于块根的形成及膨大特性，认为改善土壤通气 性能提高块根形成过程中土壤温度的日较差 ${ }^{[23]}$; 减少初 生木质部的数量 ${ }^{[15]}$ ，增强根中初生形成层的活动能力, 促进块根的形成 ${ }^{[14,16]}$ 。改善土壤通气性能提高块根中焦磷 酸化酶活性而降低淀粉酶活性 ${ }^{[15]}$; 提高块根中 ATP 含量 和 ATP 酶活性、提高脱落酸含量 ${ }^{[17]}$; 提高块根中蔗糖合 酶和 ADPG 焦磷酸化酶的活性 ${ }^{[23]}$ ，促进块根中淀粉的积
累, 促进块根的膨大。而对甘薯源库间光合产物运转差异 的分析较少，仅有部分研究认为改善土壤通气性能增加 叶片中钾、钙、锰、硼和锌的含量, 提高 ATP 含量和 ATP 酶活性, 促进叶片中光合产物的输出 ${ }^{[13,17]}$ 。而甘薯源库间 光合产物的运转需要叶、柄、茎蔓和块根共同完成, 只关 注某一个器官的生理变化, 无法确定土壤通气性引起光 合产物运转差异的关键环节。本研究结果表明, 与对照处 理相比, 疏松处理显著提高块根中 ${ }^{13} \mathrm{C}$ 同化物的输入效率, 紧实处理与之相反(图 2)。疏松处理显著提高块根中蔗糖 和淀粉含量; 显著降低地上部各器官淀粉含量, 且对茎的 作用时间早, 并显著降低茎基部蔗糖含量(表 4 和表 5)。 紧实处理显著降低块根中蔗糖和淀粉含量; 显著提高茎、 柄和叶中淀粉含量, 并显著提高茎基部蔗糖含量(表 6)。 改善土壤通气性显著降低茎基部与块根间蔗糖含量差 (表 7)。相关分析的结果也表明，茎基部与块根间蔗糖含 量差与块根熫糖和淀粉含量呈极显著负相关关系(表 8)。 说明改善土壤通气性促进甘薯源库间光合产物运转的关 键过程是光合产物由茎基部向块根的运转, 该过程运转 顺畅，源库间光合产物的运转效率高，块根中光合产物积 累多，块根产量就高。

\section{References}

[1] Rankine D R, Cohen J E, Taylor M A, Coy A D, Simpson L A, Stephenson T. Parameterizing the FAO Aquacrop model for rainfed and irrigated field-grown sweet potato. Agron J, 2015, 107: 1.

[2] Hazra P, Chattopadhyay A, Karmakar K, Dutta S. Sweet potato. In: Modern Technology in Vegetable Production. New Delhi: 
New India Publishing Agency, 2011. pp 358-370.

[3] Abdissa T A, Chali K, Tolessa F, Tadese A G. Yield and yield components of sweet potato as influenced by plant density in Adami Tulu Jido Kombolcha District, Central Rift Valley of Ethiopia. Am J Exp Agric, 2001, 1: 40-48.

[4] Mu T H, Tan S S, Xue Y L. The amino acid composition, solubility and emulsifying properties of sweet potato protein. Food Chem, 2009, 112: 1002-1005.

[5] Bourke R M. Sweet potato (Ipomoea batatas) production and research in Papua New Guinea. J Agric For Fisher, 1985, 33: 89-108.

[6] Bourke R M. Influence of soil moisture on sweet potato yield in the Papua New Guinea highlands. Mountain Res Dev, 1989, 9: 322-328.

[7] Duan W, Wang Q, Zhang H. Comparative study on carbonnitrogen metabolism and endogenous hormone contents in normal and overgrown sweetpotato. South Afr J Bot, 2018, 115: 199-207.

[8] Kazuyki W, Toshio K. Effects of the capacity and composition of soil air on the growth and yield of sweet potato plants. Jpn J Crop Sci, 1964, 33: 418-422.

[9] Anikwe M A N, Ubochi J N. Short-term changes in soil properties under tillage systems and their effect on sweet potato (Ipomea batatas L.) growth and yield in an Ultisol in south-eastern Nigeria. Aust J Soil Res, 2007, 45: 351-358.

[10] Bogunovic I, Pereira P, Kisic I, Sajko K, Sraka M. Tillage management impacts on soil compaction, erosion and crop yield in Stagnosols (Croatia). Catena, 2018, 160: 376-384.

[11] Ungureanu N, Croitoru S T, Biriş S, Voicu G, Vlă Duț V, Selvi K C. Agricultural soil compaction under the action of agricultural machinery. Actual Tasks Agric Eng, 2015, 43: 31-42.

[12] Botta G F, Tolon-Becerra A, Lastra-Bravo X, Tourn M. Tillage and traffic effects (planters and tractors) on soil compaction and soybean (Glycine max L.) yields in Argentinean pampas. Soil Tillage Res, 2010, 110: 167-174.

[13] 史春余, 王振林, 郭风法, 余松烈. 土壤通气性对甘薯养分吸 收、 ${ }^{14} \mathrm{C}$-同化物分配及产量的影响。核农学报, 2002, 16: 232-236.

Shi C Y, Wang Z L, Guo F F, Yu S L. Effects of the soil aeration on nutrient absorption, ${ }^{14} \mathrm{C}$-assimilates distribution and storage root yield in sweet potato. $J$ Nucl Agric Sci, 2002, 16: 232-236 (in Chinese with English abstract).

[14] Watanabe K, Ozaki K. Studies on the effects of soil physical conditions on the growth and yield of crop plants: III. Effects of the capacity and composition of soil air on the growth and yield of sweet potato plants. Jpn J Crop Sci, 1964, 33: 418-422.

[15] Watanabe K, Kodama T, Nomoto T. Studies on the effects of soil physical conditions on the growth and yield of crop plants: IV. Effects of the different soil structures on a few physiological characters of sweet potato plants. Jpn J Crop Sci, 1966, 34: 409-412.

[16] 王树钿, 于作庆. 甘薯在不同土壤条件下高产规律的初步研 究. 中国农业科学, 1981, 14(1): 49-55.

Wang S D, Yu Z Q. A preliminary study on the high-yielding law of sweet potato in different kind of soil. Sci Agric Sin, 1981, 14 (1): 49-55 (in Chinese with English abstract).

[17] 史春余, 王振林, 余松烈. 土壤通气性对甘薯产量的影响及其 生理机制. 中国农业科学, 2001, 34: 173-178.

Shi C Y, Wang Z L, Yu S L. Effects of soil aeration on sweet potato yield and its physiological mechanism. Sci Agric Sin, 2001, 34: 173-178 (in Chinese with English abstract).

[18] 孙向阳. 土壤学. 北京: 中国林业出版社, 2005. pp 131-136. Sun X Y. Soil Science. Beijing: China Forestry Publishing House Publishers, 2005. pp 131-136 (in Chinese).

[19] 朱伟. 葸酮-硫酸比色法测定香菇多糖含量. 北方药学, 2011, 8(8): 8-9.

Zhu W. Determination of the lentinan content by anthrone-sulfuric acid colorimetry. $J$ North Pharmacy, 2011, 8(8): 8-9 (in Chinese with English abstract).

[20] Kodama T, Nomoto T, Watanabe K. The effect of soil density and amount of fertilizer on the growth and yield. Jpn J Crop Sci, 1959, 27: 372-374.

[21] Kaoru E, Hakabu S. Effect of atmospheric humidity and soil moisture on the translocation of sucroce $-{ }^{14} \mathrm{C}$ in the sweet potato plant. Jpn J Crop Sci, 1962, 32: 41-44.

[22] Kazuyuki W, Toshio K. Effects of the different soil structures on a few physiological characters of sweet potato plants. Jpn J Crop Sci, 1965, 34: 409-412.

[23] 史文卿, 张涁彬, 柳洪鹃, 赵庆崟金, 史春余, 王新建, 司成成. 甘薯块根形成和膨大对土壤紧实度的响应机制及与产量的关 系. 作物学报, 2019, 45: 755-763.

Shi W Q, Zhang B B, Liu H J, Zhao Q X, Shi C Y, Wang X J, Si C C. Response mechanism of sweet potato storage root formation and bulking to soil compaction and its relationship with yield. Acta Agron Sin, 2019, 45: 755-763 (in Chinese with English abstract). 